\title{
Négation et opposition, même combat ? Aspects de la préfixation en non- et anti-
}

\author{
Dugas, Edwige \\ CNRS UMR STL 8163 \\ Universités Lille 1 \& Lille 3 \\ edwige.dugas@univ-lille3.fr
}

\section{Introduction}

Cet article vise à décrire un aspect du fonctionnement des noms construits sur base nominale par préfixation de non- et de anti- (dorénavant $[n o n-\mathrm{N}]_{\mathrm{N}}$ et $[a n t i-\mathrm{N}]_{\mathrm{N}}$ ) en français contemporain, tels qu'illustrés en (1) et (2) :

(1) a. Sujet : Le journal de $13 \mathrm{~h}$ de Jean-Pierre Pernod sur Tf1 un non journal?... Poser la question c'est y répondre, effectivement Jean-Pierre Pernod est un non journaliste, dans un non journal, ou le degré zéro de l'information fait pour les plus de 85 ans plus très frais psychiquement. (www)

b. Le constat, c'est que personne n'a été bon, on a fait un non-match. (www)

a. [Emile Coué] C'est une sorte d'anti Freud. Son œuvre pèse une centaine de pages et se résume à une méthode simple comme un coup de poing : répéter quarante fois, « tous les jours, à tous points de vue, je vais de mieux en mieux. » (www)

b. Pour lutter contre la pollution, la Twike, une anti-voiture suisse à énergie électrique et à pédales, a pris la bonne vieille allure futuriste d'une excentrique des années 45. (Heyna, 2013)

En (1), NON-JOURNAL, NON-JOURNALISTE et NON-MATCH sont employés dans ce que j'appelle une lecture «contraire», que je distingue de l'interprétation «ontologique » (3) et de l'interprétation « complémentaire » (4) :

(3) Une non-qualification serait un cataclysme pour l'équipe de France, qui a disputé tous les Mondiaux depuis 1997. (www)

(4) La cuisine italienne est l'une des cuisines les plus intéressantes et simples dans le monde, et est appréciée par à peu près tout le monde, jeunes et vieux, les Italiens et les non-Italiens. (www)

En (3), où NON-QUALIFICATION a une lecture ontologique, la quantification porte sur le nom base $(\mathrm{Nb})$ et le dérivé dénote l'absence de l'événement 'qualification' - autrement dit, une qualification qui n'a pas eu lieu. En (4), où NON-ITALIEN a une lecture complémentaire, le référent du $\mathrm{Nb}$ et celui du dérivé sont vus comme deux ensembles complémentaires, tous deux inclus dans un ensemble plus grand. Enfin, dans la lecture contraire, telle qu'en $(1),[n o n-\mathrm{N}]_{\mathrm{N}}$ serait un nom dont le référent ne possède pas toutes les propriétés du référent du $\mathrm{Nb}$. Intuitivement, un non-journal est un journal (télévisé, en l'occurrence) tellement mauvais qu'il n'en mérite pas le nom, parce qu'il ne remplit pas correctement son rôle, à savoir, fournir une information de qualité (pertinente, intéressante, non triviale); un non-journaliste est un mauvais journaliste qui ne mérite pas d'être appelé journaliste, par exemple parce qu'il diffuse des reportages inintéressants ; un non-match n'est pas un match digne de ce nom, c'est un match dans lequel les joueurs font une très mauvaise prestation. La négation paraît ici avoir une valeur métalinguistique.

En (2), ANTI-FREUD et ANTI-VOITURE ont une interprétation qui a notamment été qualifiée d' « antonymique » dans la littérature (Rey, 1968 ; Corbin, 1980 ; Heyna, 2008) ${ }^{1}$; c'est également le terme que je retiendrai pour désigner cette lecture des lexèmes nominaux construits par préfixation de anti- sur base nominale. Cette interprétation est à distinguer de l'interprétation « adversative » (Heyna, 
2008 ; Hathout, 2009) ${ }^{2}$, qui peut être paraphrasée par 'qui est contre la notion dénotée par $\mathrm{Nb}$ ', et où la préfixation en anti- sert à décrire une protection contre la nuisance dénotée par le $\mathrm{Nb}$, comme en (5):

$$
\text { ANTIRIDES }_{A N \mathrm{~N}} ; \text { ANTILIMACES }_{\mathrm{A} / \mathrm{N}} ; \text { ANTICAPITALISTE }_{\mathrm{AN}}
$$

À première vue, les $[n o n-\mathrm{N}]_{\mathrm{N}}$ dans leur interprétation contraire (1) et les $[a n t i-\mathrm{N}]_{\mathrm{N}}$ dans leur interprétation antonymique (2) dénotent un référent qui a certes des traits communs avec le référent du nom base, mais qui n'en possède pas toutes les caractéristiques, ou qui en possède d'autres. Ainsi, NON-HEROS (6) et ANTI-HEROS (7) semblent pouvoir s'interpréter de façon similaire :

(6) Thomas Covenant est loin d'être un héros classique, mais plutôt un non-héros qui amène le malheur sur tous les personnages qui croisent son chemin. Il refuse avec obstination le rôle de sauveur qu'on veut lui voir jouer. (www)

Le Cid, par exemple, voué à la reconquête, progresse à chaque page alors que l'anti-héros de Cervantès est contraint à la répétition du même. Aucune aventure ne le fait avancer. Le seul événement qui transforme vraiment sa destinée, c'est sa mort. (www)

On trouve également des contextes dans lesquels le dérivé en non- et celui en anti- sont présentés comme synonymes par les scripteurs, ainsi NON-STAR/ANTI-STAR, NON-MUSIQUE/ANTI-MUSIQUE en (8) et (9) :

(8) Hoffman demeure un parfait inconnu, une sorte d'anti star et hormis les Fan des Padres, qui se soucie de ce gars? Joueur à la personnalité bien peu charismatique, Hoffman a eu une carrière des plus anonymes, passée dans des clubs peu flashy. (...) Enfin, pour finir sa carrière de "non star", il ira vers un autre club plus wild et plus sauvage, mais qui n'intéresse là encore que peu de gens, les Brewers de Milwaukee, où l'on a senti que Hoffman était sur la fin. (www)

(9) L'œuvre musicale de Haydn, (...) c'est la musique non dramatique par excellence écrite à une époque où l'on cherchait justement le drame. Une "non-musique, une anti musique, une musique qui renonce aux besoins de divertir que la nouvelle société bourgeoise exige de ses héros musiciens : des effets surprenants, beaucoup de divertissement, des gestes empruntés à l'Opéra, et surtout des aspects drôles et étincelants de l'opéra bouffe. Il n'y a pratiquement rien de cela dans Sieben Letzte Worte (...) ». (www)

M'appuyant sur un corpus de formes attestées dans le Trésor de la Langue Française informatisé (TLFi) ${ }^{3}$, Frantext $(\mathrm{F})^{4}$ et la Toile (www), je cherche dans cet article à comparer l'interprétation contraire des [non$\mathrm{N}]_{\mathrm{N}}$ et l'interprétation antonymique des $[a n t i-\mathrm{N}]_{\mathrm{N}}$. Je m'interrogerai sur les éventuelles contraintes portant sur les bases, sur la relation sémantique entre le $\mathrm{Nb}$ et le lexème construit ainsi que sur le sens des dérivés ; ma principale conclusion sera que ces deux interprétations ne sont pas équivalentes, non- étant spécialisé dans l'expression de la négation, anti- dans l'expression de l'opposition.

L'article est organisé comme suit. La deuxième partie est consacrée à un état de l'art de l'interprétation contraire des $[n o n-\mathrm{N}]_{\mathrm{N}}$ et de l'interprétation antonymique des $[a n t i-\mathrm{N}]_{\mathrm{N}}(\S 2)$. Puis j'expose dans une troisième partie la méthodologie adoptée pour la constitution du corpus de travail et l'annotation des données (\$3). Je présente ensuite les résultats de l'annotation de mon corpus, et je propose, à la lumière des données collectées, une analyse comparée des deux interprétations (\$4). Je termine par quelques remarques conclusives.

\section{2 État de l'art}

Cette section commence par quelques rappels théoriques (\$2.1). La suite est consacrée tout d'abord à un état de l'art, axé sur un rapprochement des $[n o n-\mathrm{N}]_{\mathrm{N}}$ dans leur interprétation contraire avec les $[n o n-\mathrm{N}]_{\mathrm{N}}$ et les $[u n-\mathrm{N}]_{\mathrm{N}}$ de l'anglais (§2.2), puis par un état de l'art des $[a n t i-\mathrm{N}]_{\mathrm{N}}$ antonymiques $(\S 2.3)$. Y sont suggérés des points communs aux deux interprétations : les préfixations en non- et en anti- touchent certaines propriétés attachées aux référents des $\mathrm{Nb}$, ce qui a pour effet de remettre en question l'appartenance du référent du nom dérivé à la catégorie dénotée par le $\mathrm{Nb}$. Cet état de l'art permet également de souligner la valeur fondamentalement oppositive de la préfixation en anti-. 


\subsection{Quelques précisions théoriques}

\subsubsection{Contraires et antonymes}

Une façon assez simple d'appréhender la notion de contraire est de le faire en la mettant en parallèle avec celle de contradictoire. Contraire et contradictoire sont les termes employés par Horn (1989: 69) à partir des classifications faites par Aristote. Cette distinction est parfois reprise sous d'autres termes, certains auteurs préférant parler d'antonymes (pour les contraires) et de complémentaires (pour les contradictoires) (Cruse, 1986 : 197sqq. ; Lyons, 1977 : 279). Dans ce travail, je fais le choix de parler de contraires et de complémentaires. Les contraires sont gouvernés par la loi de non-contradiction, tandis que les complémentaires sont gouvernés et par la loi de non-contradiction et par la loi du tiers exclu (Horn, 1989 : 270 sq.). Cela signifie que, dans une relation contraire, les deux termes peuvent être vrais ensemble, mais ne peuvent pas être faux ensemble, tandis que dans une relation de complémentarité, les termes ne peuvent être ni tous les deux vrais ni tous les deux faux : l'un est nécessairement vrai et l'autre nécessairement faux. Ainsi, deux termes contraires «représentent des aires situées aux pôles opposés d'une dimension donnée et laissent une place pour d'autres possibilités entre eux » ${ }^{5}$ (Zimmer, 1964: 21), et deux termes complémentaires «divisent [entre eux] de façon exhaustive un domaine conceptuel en deux compartiments mutuellement exclusifs, de telle sorte que ce qui n'appartient pas à l'un des compartiments appartient nécessairement à l'autre $»^{6}$ (Cruse, 1986 : 198). Le nom NON-ITALIEN en (4) est un complémentaire : l'ensemble des individus appréciant la cuisine italienne est constitué de l'ensemble des Italiens et de l'ensemble des non-Italiens, et chaque individu appartient nécessairement à l'un ou l'autre de ces deux ensembles. Le nom NON-JOURNALISTE en (1), comme tous les [non-N $]_{\mathrm{N}}$ étudiés dans cet article, est un exemple de contraire, puisqu'un journaliste peut être un non-journaliste à plusieurs degrés : il peut être un mauvais journaliste, un très mauvais journaliste, etc. De plus, il peut être un nonjournaliste pour des raisons différentes : parce qu'il diffuse des reportages inintéressants, parce qu'il divulgue ses sources, parce qu'il ne sait pas lire un prompteur, etc. (Dugas, 2012).

\subsubsection{Prototypes et stéréotypes}

Dans les approches structuralistes du sens et des catégories, la signification lexicale est décomposable en traits sémantiques (marqueurs, sèmes) qui ont pour particularité d'être en nombre fini, d'être discrets, et de fonctionner généralement en opposition. D'autre part, les propriétés nécessaires et suffisantes constituent la signification, qui permet d'identifier le référent (cf. Fradin, 1984). Étant donné que les catégories ont des frontières délimitées, un objet soit appartient à une catégorie, soit n'y appartient pas. Pour appartenir à une catégorie, un objet doit posséder les attributs définitoires de cette catégorie : cela signifie que les membres d'une même catégorie ont un statut catégoriel égal (cf. entre autres Kleiber, 1990 : 22). En général, ces attributs sont des «traits » affectés d'une valeur positive ou négative. Un exemple classique est celui de chaise (Katz, 1972 : 259 ; Pottier, 1965) :

(10) Chaise : /+objet concret/, /+portable/, /+dossier/,/+pieds/,/- bras/,/+ pour s'asseoir/

La nature des traits ou leur forme (qu'il s'agisse d'une proposition infinitive 'pour s'asseoir'; substantif 'dossier', 'pieds', 'objet concret'; adjectif 'portable') n'importe pas, tous les traits ont un statut égal, ils participent tous à la signification (la signification est la somme de ces traits), et n'importe quoi peut être employé comme trait. Il s'agit donc d'un modèle componentiel, puisque la signification s'obtient par addition des traits.

Avec les cognitivistes et les tenants de l'approche en terme de prototypie (e.a. Rosch, 1978), je défends au contraire l'idée qu'au sein d'une catégorie, certains individus (au sens large du terme) sont de meilleurs exemplaires de la catégorie que d'autres et qu'il existe des individus dont l'appartenance à la catégorie est incertaine : les catégories ont des frontières floues. Les caractéristiques des catégories prototypiques sont différentes des catégories des structuralistes. En effet, les catégories prototypiques (i) ne sont pas définies par un ensemble d'attributs nécessaires et suffisants, (ii) ont une structure du type « ressemblance de famille $»^{7}$ : leur structure sémantique est faite groupes de significations qui se recoupent partiellement, 
(iii) sont caractérisées par une appartenance catégorielle graduelle, des degrés variables de représentativité : les membres de la catégories ne le sont pas au même degré, ils peuvent être plus ou moins représentatifs de la catégorie, (iv) ont des limites floues, non définies.

D'autre part, l'examen des données de mon corpus, on le verra, suggère une analyse non pas en termes de propriétés prototypiques, mais de propriétés stéréotypiques. Geeraerts exprime la distinction entre prototype et stéréotype de la manière suivante : «les stéréotypes sont les prototypes pris d'un point de vue social $»^{8}$ (Geeraerts, $2008: 26$ ). Comme le rappelle Geeraerts (2006), stéréotypes et prototypes ont en commun de mettre en jeu les informations sémantiques qui sont saillantes dans une catégorie mais non suffisantes pour définir la catégorie dans son entier; de plus, il est fréquent (mais pas systématique) que les caractéristiques appartenant au stéréotype correspondent aux caractéristiques du prototype. Mais le prototype est avant tout une notion psychologique, qui concerne l'organisation cognitive de la connaissance et de la signification, et semble postuler l'homogénéité de la communauté linguistique (e.a. Putnam, 1975 ; Geeraerts, 2008). Au contraire, le stéréotype est « un ensemble minimum de données socialement déterminées concernant l'extension de la catégorie »" (Geeraerts, $2008: 26)$ : il s'agit donc plutôt d'une notion sociolinguistique, qui constitue ce que l'individu est supposé savoir sur les référents des catégories qu'il mobilise, selon le principe de la « division du travail linguistique ${ }^{10}$; il est attaché à un contexte et à un groupe socio-culturel donné, une "communauté linguistique ». La notion de communauté linguistique, en lien avec la notion de stéréotype, remonte à Putnam (1975). Je reprends ici la définition donnée par Anscombre : elle désigne «tout ensemble de sujets parlants qui est présenté comme partageant (entre autres choses) une certaine liste de termes affectés des mêmes significations » (Anscombre, 2001 : 57). Ainsi, «un même sujet parlant fera ainsi partie de diverses communautés linguistiques à des moments différents où il est locuteur (...) Tout locuteur, lorsqu'il parle, i.e. dans son rôle de locuteur, parle en tant que membre d'une certaine communauté linguistique » (Anscombre, 2001 : 58). En bref, je conçois donc le stéréotype comme un ensemble non fermé de propriétés associées à un nom ; ces propriétés ne correspondent pas nécessairement à la réalité (elles peuvent être fausses ou contradictoires).

\subsection{L'interprétation contraire des [non- $\mathrm{N}]_{\mathrm{N}}$ : rapprochement avec les données de l'anglais}

La littérature est pratiquement muette sur l'interprétation des dérivés nominaux en non- en français, et ce pour plusieurs raisons. Premièrement, le statut de non-, quand il est employé de façon liée (et non pas comme adverbe de phrase, en syntaxe, p. ex. Non, cet homme n'est pas un bon journaliste) est problématique et différentes propositions ont été faites à son sujet : mot de négation (Gaatone, 1971), adverbe négatif (Huot, 2007), morphème libre (Di Sciullo \& Tremblay, 1993), préfixe (Zimmer, 1964 ; Kalik, 1971 ; Anscombre, 1994 ; Dal et al., 2007). Deuxièmement, l'essentiel des travaux touchant à nondans son emploi lié portent sur les cas où il s'adjoint à un adjectif (p. ex. non occupé, non accessible, non remboursable, non vénéneux), où les auteurs s'intéressent généralement aux similarités de ces adjectifs avec ceux préfixés par in- (p. ex. inoccupé, inaccessible). Par ailleurs, lorsque les $[\text { non- } \mathrm{N}]_{\mathrm{N}}$ sont mentionnés, ils le sont conjointement aux formes adjectivales, alors que ces deux types de formes gagneraient à être examinés séparément, dans la mesure où les adjectifs "préfixés " par non- ont un comportement plutôt syntaxique (p. ex. les tours typiquement syntaxiques comme des locaux non occupés par les militaires, une zone non encore accessible, un point de vue peu ou non compris, un billet non remboursable ni échangeable) (Dugas, 2013). De fait, les arguments et exemples proposés dans la littérature pour les $[n o n-\mathrm{N}]_{\mathrm{N}}$ dans l'interprétation que j'ai appelée «contraire » sont quasiment inexistants.

Les travaux sur le préfixe non- et le préfixe un- en anglais fournissent toutefois des pistes intéressantes. Algeo (1971), notamment repris par Bauer (1983) et par Plag (2003), distingue trois préfixes non- : le non- qu'il appelle «privatif » (11), le non- «péjoratif » (12), et le non- « dissimulatif » (13) :

(11) We accept war news with the same nonthought as the morning cup of coffee. 'Nous acceptons la guerre avec la même non-pensée que notre tasse de café du matin.' (Algeo, $1971: 90$ ) 
(12) noncandidate: one who is declared candidate but whose election is so unlikely that he can be set aside as a possible contender. 'non-candidat: personne déclarée candidate mais dont l'élection est si improbable qu'elle ne peut pas être considérée comme un possible concurrent.' (Algeo, 1971 : 94 sq.)

(13) The grass is usually greener in a hippie house. Sans Souci Temple, near downtown Los Angeles, accommodates a happy non-family of 24 people, including these adults and child-none of them related to one another. 'En général, l'herbe est plus verte dans une maison hippie. Sans Souci Temple, près de Los Angeles, est le lieu de résidence d'une heureuse non-famille de 24 personnes, incluant des adultes et des enfants qui n'ont aucun lien de parenté.' (Algeo, 1971 : 94 sq.)

Dans son emploi «privatif» (10), le préfixe non- permet d'exprimer l'absence du référent dénoté par le nom base, qui se présente comme objective, sans qu'aucun jugement de valeur ne soit porté. Ce type d'emploi correspond à l'interprétation que j'ai qualifiée d'ontologique (3) et ne nous intéresse pas ici. Par contre, le non- "péjoratif» (12) et le non- "dissimulatif» (13) sont a priori très proches de l'interprétation contraire des $[\text { non-N }]_{\mathrm{N}}$ en français, dans la mesure où il semble être question de propriétés qui ne correspondent pas tout à fait au stéréotype, de propriétés qui ne sont pas tout à fait celles auxquelles on peut s'attendre. La différence que fait Algeo entre ces deux lectures est la suivante :

(i) La fonction du non- péjoratif est de porter un jugement négatif sur la «valeur » du référent dénoté par le $\mathrm{Nb}$. Le référent dénoté par le dérivé possède «les accidents, mais pas la substance »"

(ii) Avec le non- dissimulatif, le référent dénoté par le dérivé «possède la valeur, mais pas les caractéristiques de surface ou l'identité reconnue » du référent dénoté par le $\mathrm{Nb}$; le référent possède «la substance mais pas les accidents ${ }^{12}$ (Algeo, $\left.1971: 95\right)$.

L'analyse proposée par Algeo est intéressante mais ne dit pas grand-chose du type de propriétés affectées par la préfixation en non-. Les notions de substance et d'accident, issues de la Métaphysique aristotélicienne, renvoient respectivement aux parties immanentes des êtres, « dont la destruction serait la destruction du tout » (2008: 195 / livre D, 8) et à «ce qui est la propriété de quelque chose et qu'il est vrai de dire, mais qui pourtant ne l'est ni nécessairement ni la plupart du temps » (1998: 222 / livre D, 30 ) : la substance est essentielle, l'accident ne l'est pas. Algeo ne dit pratiquement rien non plus sur la relation sémantique qui unit le $\mathrm{Nb}$ et le $\mathrm{N}$ dérivé. Le problème est que si l'on suit son analyse, les [non$\mathrm{N}]_{\mathrm{N}}$ péjoratifs devraient se caractériser par une absence d'inclusion sémantique entre le $\mathrm{Nb}$ et le $\mathrm{N}$ dérivé, puisque le référent possède seulement les accidents ; or en (12), le non-candidat est bien un candidat. De même, les $[n o n-\mathrm{N}]_{\mathrm{N}}$ avec non- dissimulatif devraient se caractériser par une inclusion sémantique entre le $\mathrm{Nb}$ et le $\mathrm{N}$ dérivé, puisque la substance est conservée, mais la non-famille décrite en (13) n'est pas une famille. Les autres exemples que donne Algeo dans son article ne sont pas plus convaincants, et il ne propose aucun critère pour discriminer les cas où l'on a affaire à une interprétation péjorative, et ceux où il s'agit d'une interprétation dissimulative.

Horn (2002a, 2002b) propose une analyse du préfixe anglais un- sur bases nominales, que je mentionne ici étant donné que l'interprétation de ces dérivés paraît très similaire à l'interprétation contraire des [non$\mathrm{N}]_{\mathrm{N}}$ en français. Il s'appuie sur la notion de protoype et identifie deux interprétations différentes pour les un-N, qu'il appelle « classe A » (pour almost 'presque') et « classe B » (pour barely 'à peine') :

(i) Les $u n-\mathrm{N}$ de la classe $\mathrm{A}$ « ne satisfont pas les critères structurels pour la catégorie $\mathrm{X}$ mais partagent sa fonction, évoquant une catégorie plus grande englobant à la fois $\mathrm{X}$ et $u n-\mathrm{X} »^{13}$ (Horn, 2002b : 65). Le référent d'un un- $\mathrm{N}$ est presque un membre de la catégorie $\mathrm{N}$ - il n'est donc pas un membre de cette catégorie - comme l'illustre l'exemple de UNTURKEY 'non-dinde' en (14) :

(14) unturkey: used for mock turkey breasts made from soy sold at natural foods store. 'non-dinde : employé pour désigner des imitations de blanc de dinde au soja vendus dans les magasins de produits biologiques.' (Horn, 2002b : 66) 
(ii) Dans le cas des $u n-\mathrm{N}$ de la classe $\mathrm{B}$, «[le référent] est un membre de la catégorie (...) mais un membre périphérique ou non prototypique (...), un membre auquel il manque une propriété fonctionnellement significative (mais pas nécessaire) de la catégorie en question $»^{14}$ (Horn, 2002b : 66). Le référent dénoté par le un- $\mathrm{N}$ est certes à peine un membre de la catégorie $\mathrm{N}$, mais il est bien un membre de cette catégorie - comme l'illustre UNWOMAN 'non-femme' en (15) :

(15) unwomen: label for women in Margaret Atwood's The Handmaid's Tale (1985) who, because they are not fertile, are shipped to the colonies as slave labor. 'non-femmes: dans The Handmaid's Tale (1985) de Margaret Atwood, nom donné aux femmes qui, en raison de leur infertilité, sont envoyées comme esclaves dans les colonies.' (Horn, 2002b : 66)

Comme dans l'analyse proposée par Algeo pour les $[n o n-\mathrm{N}]_{\mathrm{N}}$, les $u n-\mathrm{N}$ peuvent, pour Horn, être classés en deux groupes, selon que leur référent est ou non un membre de la catégorie. Plus précisément, l'analyse de Horn suggère que les $u n-\mathrm{N}$ de la classe A étendent en quelque sorte l'extension de la catégorie, à la manière d'une enclosure. Le terme d'enclosure est la traduction proposée par Kleiber \& Riegel (1978) du terme hedge introduit par Lakoff pour désigner les « mots dont le sens contient un vague implicite » et «dont le rôle est de rendre les choses plus floues ou moins floues » ${ }^{15}$ (Lakoff, $1973: 474$; voir aussi Kleiber \& Riegel, 1978). L'idée sous-jacente est que l'appartenance catégorielle est une question de degré. Ainsi, sorte de, presque, plutôt sont des enclosures qui étendent la dénotation du mot sur lequel elles portent, tandis que vrai, littéralement, par excellence restreignent la dénotation aux référents les plus prototypiques de la catégorie.

Pour résumer, l'analyse d'Algeo (1971) et Horn (2002a, 2002b) est centrée sur l'idée que les préfixations en non- et un- affectent le degré d'appartenance du référent du nom construit à une catégorie du nom base. Tandis qu'Algeo mobilise les concepts artistotéliciens de substance et d'accident, Horn fait appel à la notion de prototype. Ces deux approches ne me paraissent pas adéquates pour rendre compte de ce type de dérivés - du moins en français - et, comme annoncé, je parlerai dans cet article de propriétés stéréotypiques. Par contre, il faut souligner que d'une part, Algeo et Horn suggèrent que les $[n o n-\mathrm{N}]_{\mathrm{N}}$ et les $u n-\mathrm{N}$ ont un sens axiologique; d'autre part, ils semblent considérer que ces dérivés ne sont pas des dénominations - constats que l'on peut semble-t-il étendre au français (§4.2.1).

\subsection{L'interprétation antonymique des [anti-N $]_{\mathrm{N}}$}

\subsubsection{Anti- annule les propriétés attachées au référent du $\mathrm{Nb}$}

La littérature est assez riche sur l'interprétation antonymique des $[\text { anti- } \mathrm{N}]_{\mathrm{N}}$ en français, bien que les travaux sur anti- aient plus souvent porté sur l'interprétation adversative. Les auteurs s'accordent à dire que, dans l'interprétation antonymique des $[a n t i-\mathrm{N}]_{\mathrm{N}}$, la préfixation en anti- entraîne une annulation (ou inversion, renversement) des propriétés attachées au référent $\mathrm{du} \mathrm{Nb}$. Ils diffèrent toutefois quant au nombre et à la nature des propriétés affectées par la préfixation : pour certains, toutes les propriétés sont annulées (Rey, 1968 ; Corbin, 1980 ; Durand, 1982) ; pour d'autres, il s'agit des propriétés subjectives (Zribi-Hertz, 1973), des propriétés stéréotypiques (Fradin, 1997a, 1997b), des propriétés (proto)typiques (Heyna, 2008 ; Hathout 2009), ou encore du trait « vrai » (Heyna, 2013).

Rey propose une analyse componentielle en termes de traits (cf. §2.1.2); les «traits pertinents » sont inversés par anti-, tandis que la structure sémique reste identique (Rey, $1968: 53$ ). La représentation d'un $[\text { anti-N }]_{\mathrm{N}}$ sera alors la suivante :

$$
\begin{aligned}
& \mathrm{X}=\mathrm{S} 1, \mathrm{~S} 2, \mathrm{~S} 3 \ldots \mathrm{Sn} \text { (traits pertinents positifs) } \\
& \text { Anti-X = -S1, -S2, -S3 .. -Sn (mêmes traits négativisés) }
\end{aligned}
$$

Rey prend l'exemple des Antimémoires de Malraux : les traits propres au référent mémoires sont inversés, mais les Antimémoires «se présentent comme une sous-espèce de Mémoires (même structure sémique: même incluant logique, même classème 'objet', 'livre') » (Rey, $1968: 55)$. L'auteur ajoute que « ce sont à la fois des mémoires et des non-mémoires » (ibid.), ce qui pourrait suggérer une équivalence entre les 
deux types de dérivés. Malheureusement, il ne dit pas ce que sont ces «traits pertinents » et ce qui les distinguerait de traits non pertinents, ni à partir de quand, pour reprendre l'exemple, des Antimémoires ne sont plus des mémoires (d'autre part, les classèmes 'objet' et 'livre' ne sont pas suffisants pour distinguer des mémoires d'un autre type de livre).

Les analyses de Corbin (1980) et de Durand (1982) s'inscrivent dans autre un cadre théorique mais ont en commun avec celles de Rey de considérer que (i) l'ensemble des propriétés est affecté par la préfixation en anti- : l' $[\text { anti-N }]_{\mathrm{N}}$ antonymique et le $\mathrm{Nb}$ désignent des référents aux caractéristiques opposées, (ii) le dérivé et la base sont dans une relation d'inclusion sémantique (des antimémoires sont des mémoires).

L'analyse de Fradin (1997a, 1997b) est différente. Selon lui, le N dérivé « dénote un objet caractérisé par le fait qu'il se définit par la négation des propriétés stéréotypiquement attachées à N » (Fradin, 1997a : 90). Ainsi, anti- «n'opère pas sur la représentation sémantique du N prise en bloc mais au contraire sur chaque propriété qu'encode cette dernière » (Fradin, 1997b : 340). Fradin fait la distinction entre les propriétés constitutives et les propriétés stéréotypiques: anti- opère seulement sur les propriétés stéréotypiques. Par exemple, dans ANTI-MADONNA, employé dans l'énoncé Céline Dion, l'anti-Madonna, anti- annule les propriétés stéréotypiques 'provocatrice', 'femme-fatale', tout en maintenant les propriétés constitutives 'femme' et 'chanteuse' du Nb (Fradin, 1997b : 340). Toutefois, ceci suppose que l'on se soit accordé, au préalable, sur les propriétés stéréotypiques attachées au référent du $\mathrm{Nb}$. C'est ce que souligne notamment Zribi-Hertz, pour qui les $[\text { anti-N }]_{\mathrm{N}}$ antonymiques sont paraphrasables par « (tout) le contraire de » (Zribi-Hertz, 1973 : 48) et qui considère que dans cette interprétation, le rôle sémantique du préfixe anti- est d'exprimer non pas le contraire « objectif» du Nb, mais son « contraire subjectif»; de plus, les propriétés attachées au Nb peuvent varier « à l'infini selon les locuteurs » (Zribi-Hertz, 1973: 49).

Heyna $(2008,2009,2013)$ et Hathout (2009) adoptent une position proche de celle de Fradin (1997a, $1997 b$ ) et considèrent que le préfixe anti- est un opérateur qui agit sur l'intension du $\mathrm{Nb}$ en annulant ou en renversant certains des traits sémantiques considérés comme (proto)typiques. Le référent appartient à la catégorie des $\mathrm{Nb}$, parce que «suffisamment de traits pertinents sont maintenus ». Par exemple, « un antihéros reste malgré tout une sorte de héros » (Heyna, 2008: 13 sq.). Les auteurs soulignent toutefois que les traits négativisés ne sont pas toujours identifiables, et que la glose accompagnant l'[anti$\mathrm{N}]_{\mathrm{N}}$ permet d'identifier les propriétés annulées.

Il ressort donc de la littérature le constat d'une double relation entre le dérivé et sa base : le nom dérivé est à la fois dans une relation sémantique d'antonymie et d'hypo/hyperonymie avec son nom base. Pour Heyna $(2008,2009,2013)$ et Hathout $(2009)$, les $[a n t i \mathrm{~N}]_{\mathrm{N}}$ appartiennent à la classe du $\mathrm{Nb}$ et en forment une sous-classe, donc il y a rapport d'inclusion logique de type hypo/hyperonyme ; mais ils expriment également un écart par rapport au prototype exprimé par le $\mathrm{Nb}$, d'où un rapport d'antonymie. Hathout et al. (2004) prennent l'exemple du lexème AFFICHE tel qu'illustré en (17) et analysent ANTI-AFFICHE comme un co-hyponyme, avec « affiche normale », de « affiche» :

(17) Cette affiche de saison est presque une anti-affiche, sombre, sourde, discrète mais une image qui a du sens. (Hathout et al., $2004: 9$ )

Appliquée à l'exemple (17), l'hypothèse de Heyna (2013) selon laquelle anti- opère sur le trait « vrai » signifie que l'on aurait $\mathrm{AFFICHE}_{1}$ qui serait l'hyperonyme, AFFICHE 2 l'hyponyme non marqué, correspondant à une « vraie » affiche, et ANTI-AFFICHE l'hyponyme marqué, où anti- porterait sur le trait « vrai » de $\mathrm{AFFICHE}_{2}$ :

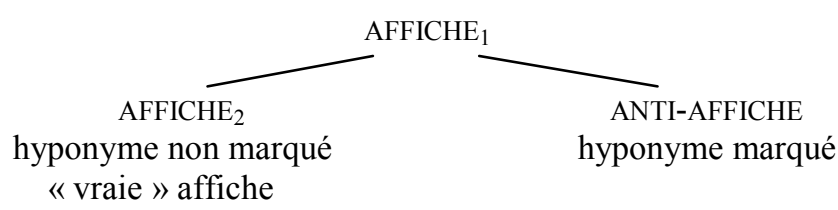


Donc selon cette approche, le lexème AFFICHE est «polysémique »(Heyna, 2013: 66) : il peut désigner l'hyperonyme (n'importe quel type d'affiche), ou l'hyponyme non marqué (une «vraie » affiche). Pour mémoire, la distinction faite par Lyons (1977) entre lexème sémantiquement marqué et lexème sémantiquement non marqué est que le premier a un sens plus spécifique que le lexème sémantiquement non marqué correspondant (Lyons, 1977 : 307) (cf. également Cruse, 1986 : 256). Par exemple, lionne a un sens plus spécifique que lion, qui peut désigner soit l'espèce, soit l'animal mâle : dans certains contextes, la différence entre lionne et lion est neutralisée, et dans d'autres, il n'est pas possible d'utiliser le terme non marqué (lion) pour référer au terme marqué (lionne). Mais dire d'un lexème qu'il est sémantiquement non marqué n'équivaut pas à dire qu'il est polysémique.

L'analyse de Fradin (1997a, 1997b) est intéressante parce qu'elle mobilise la notion de stéréotype, qui est plus appropriée pour rendre compte des données de mon corpus que celle de Rey (1968), qui raisonne en termes de catégories nécessaires et suffisantes, ou que celles d'Heyna $(2008,2009,2013)$ et de Hathout (2009) en termes de prototype. Quant à la relation sémantique qui lie le nom base au nom dérivé, mêlant, selon la plupart des auteurs, antonymie et hypo/hyperonymie, elle reste à vérifier.

\subsubsection{Anti- exprime une opposition}

J'ai mentionné à plusieurs reprises que le préfixe anti- sert également à former des dérivés dont on dira qu'ils ont une valeur adversative, ou d'opposition: le dérivé a le sens de 'qui cherche à combattre la

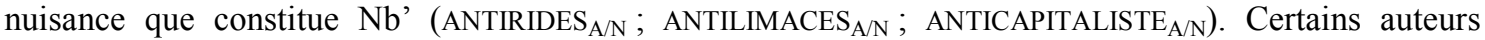
(Zribi-Hertz, 1973 ; Hathout, 2009) considèrent qu'au-delà des différences sémantiques entre les [anti $\mathrm{N}]_{\mathrm{N}}$ adversatifs et les [ant $i \mathrm{~N}]_{\mathrm{N}}$ antonymiques, le préfixe anti- permet toujours de référer à une opposition. Plus précisément, le sens le plus saillant des lexèmes préfixés en anti- serait un sens adversatif, oppositionnel, et le sens de négation serait secondaire. Cette "unité de famille » (selon l'expression employée par Hathout, 2009) est peut-être avant tout à rechercher dans l'étymologie du préfixe anti-. Le préfixe anti-

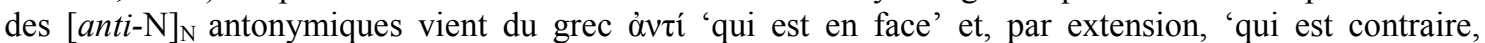
opposé' (TLFi, s.v. anti-) ${ }^{16}$.

Comme le souligne Zribi-Hertz (1973), les deux valeurs distinctes (c'est-à-dire l'interprétation adversative et l'interprétation antonymique) des dérivés en anti- «ont en commun la notion d'opposition» (Zribi-Hertz, 1973: 20), qui se traduit soit par l'idée d'antagonisme (pour les dérivés adversatifs), soit par l'idée de contraire (pour les dérivés antonymiques). Comme tous les auteurs à sa suite, Zribi-Hertz constate que la valeur adversative est la plus fréquemment présente dans les dérivés en anti- ; c'est également un constat que j'ai pu faire lors de la constitution de mon corpus de travail.

On notera aussi que le poids sémantique d'anti- est tel qu'il est possible de lui attribuer un sens hors contexte, puisqu'on le rencontre facilement en emploi autonome dans le discours, tout comme son antonyme pro- $^{-17}$; dans cet emploi, anti- exprime l'opposition :

a. Sur le non-cumul, pro et anti jouent la surenchère. (Wiktionary, s.v. anti)

b. Mariage gay : la mobilisation des antis ne faiblit pas. (Wiktionary, s.v. anti)

On remarquera que le préfixe non- ne possède pas cet emploi autonome $\left({ }^{? ?}\right.$ Sur le non-cumul, oui et non jouent la surenchère /?"Mariage gay : la mobilisation des non ne faiblit pas).

A aussi été soulignée l'existence de dérivés polysémiques qui, selon le contexte, peuvent avoir les interprétations adversative et antonymique. Enfin, certains dérivés peuvent avoir les deux interprétations en même temps, par exemple ANTIPAPE désigne à la fois « un pape dont les propriétés caractérisantes sont inversées puisqu'il n'appartient pas à la papauté catholique romaine» et « une personne qui considère cette papauté comme une nuisance, qui s'oppose à elle et en conteste la légitimité ; ANTIPEDAGOGIE désigne à la fois «une pédagogie basée sur des principes différents de ceux de la pédagogie traditionnelle » et qui «considère (...) cette dernière comme une nuisance, la combat et cherche à s'imposer à sa place » (Hathout, 2009: 105). Dans le même ordre d'idées, Zribi-Hertz (1973) remarque 
que « les deux valeurs en question [adversative et antonymique] ne se distinguent pas toujours de manière très tranchée, et peuvent même se mêler dans l'interprétation » (Zribi-Hertz, 1973 : 52).

\section{Méthodologie}

\subsection{Corpus de travail}

Afin de disposer de données issues de divers domaines et registres de langue, j'ai construit mon corpus à partir de trois sources : (i) la nomenclature du TLFi, (ii) les textes de 1900 à 2012 contenus dans Frantext, (iii) la Toile via l'outil de veille GlossaNet ${ }^{18}$ et le corpus COLiBrI (Schäfer \& Bildhauer, 2012) ${ }^{19}$. L'apprêtement des données s'est fait différemment pour les $[\text { non-N }]_{\mathrm{N}}$ et pour les $[\text { anti- } \mathrm{N}]_{\mathrm{N}}$, mais dans les deux cas, manuellement et en contexte.

Concernant les $[n o n-\mathrm{N}]_{\mathrm{N}}$, la difficulté vient du fait que le préfixe non- a pour homonyme l'adverbe de phrase non, ce qui entraîne une quantité très importante de bruit lors de l'interrogation des corpus et un gros travail de tri. D'autre part, le préfixe non- ne s'orthographie pas nécessairement avec un trait d'union $^{20}$, ce dont il faut tenir compte dans la formulation des requêtes. La première étape a donc été de mettre de côté, d'une part, toutes les occurrences de non qui étaient des formes syntaxiques (Non, cet homme n'est pas un bon journaliste), ainsi que les très rares cas où non précède un verbe (Les aveugles " non voient ", les armements "non prolifèrent»), et d'autre part, les occurrences de non devant un adjectif (non remboursable), ou devant un adverbe précédant un adjectif (des ponts non encore terminés). Les premiers cas de figure sont majoritaires, concernant environ $89 \%$ des occurrences de non; les occurrences de non devant un adjectif se rencontrent dans une proportion de $8 \%$ du total des occurrences; les $3 \%$ restants sont des $[\text { non-N }]_{\mathrm{N}}$.

La seconde étape a consisté à classer les $[n o n-\mathrm{N}]_{\mathrm{N}}$ dans une des interprétations mentionnées en introduction, à savoir l'interprétation contraire (1), ontologique (3) et complémentaire (4). En moyenne, les trois interprétations sont réparties comme suit: $5 \%$ de $[n o n-\mathrm{N}]_{\mathrm{N}}$ contraires, $68 \%$ de $[\text { non-N }]_{\mathrm{N}}$ ontologiques et $27 \%$ de $[n o n-\mathrm{N}]_{\mathrm{N}}$ complémentaires. Il faut tout de même signaler que l'attribution de l'interprétation n'est pas toujours aisée : la moitié des $[n o n-\mathrm{N}]_{\mathrm{N}}$ contraires retenus sont à la frontière entre une interprétation contraire et une interprétation ontologique, ou entre une interprétation contraire et une interprétation complémentaire.

Le travail de tri est facilité pour les $[a n t i-\mathrm{N}]_{\mathrm{N}}$, étant donné que le préfixe anti- n'a pas de correspondant ou d'homonyme non préfixal. Par contre, il faut là aussi tenir compte du fait que les dérivés peuvent être orthographiés avec ou sans trait d'union. La première étape a consisté à mettre de côté les cas où l'output de la préfixation en anti- était un adjectif (c'est le cerveau le plus anti-poétique que je connaisse; le précurseur génial, hardi, anti-bourgeois, anti-capitaliste et anti-parlementaire, du fascisme moderne), soit environ $64 \%$ des cas. Les $36 \%$ restants sont donc des $[a n t i-\mathrm{N}]_{\mathrm{N}}$.

Les $[\text { anti-N }]_{\mathrm{N}}$ ont ensuite été classés selon leur interprétation. J'ai d'emblée mis de côté l'interprétation spatiale, étant donné qu'elle correspond au préfixe latin ante- (cf. \$2.3.2 et note 16). J'ai ensuite distingué l'interprétation adversative (les odeurs profondes de l'anti-mite et du parfum qui foudroie), l'interprétation que j'ai appelée "d'inversion", et qui concerne des $[a n t i-\mathrm{N}]_{\mathrm{N}}$ employés dans le vocabulaire de la physique des particules (anti-fermion, anti-quark) ou de la stylistique (antibachée, antimétabole) $^{21}$, et enfin, l'interprétation antonymique (par exemple (2)). En moyenne, les trois interprétations sont réparties comme suit : 49,3\% d'[anti-N $]_{\mathrm{N}}$ à interprétation adversative, $48,5 \%$ à interprétation antonymique, et $2,2 \%$ à interprétation d'inversion. Comme pour les $[\text { non- } \mathrm{N}]_{\mathrm{N}}, 1$ 'attribution d'une interprétation a parfois posé problème : la moitié des $[\text { anti- } \mathrm{N}]_{\mathrm{N}}$ à interprétation antonymique se situent entre une interprétation antonymique et une interprétation adversative. Ceci rejoint le constat fait par Zribi-Hertz (1973) et mentionné plus haut d'une difficulté à discriminer, dans certains cas, les deux interprétations. Des exemples sont donnés au §4.2.2. 
On notera en passant que ces deux types de dérivés sont très peu fréquents et qu'il est nécessaire de brasser un grand nombre de données afin d'obtenir un échantillon suffisant pour pouvoir proposer une analyse. À l'issue du travail de tri ont été retenus 127 types (pour 215 tokens) de $[\text { non-N }]_{\mathrm{N}}$ à lecture contraire et 166 types (pour 382 tokens) d'[anti-N $]_{\mathrm{N}}$ à lecture antonymique.

\subsection{Paramètres d'annotation}

Un rapide examen du corpus suggère que les $[\text { non }-\mathrm{N}]_{\mathrm{N}}$ contraires et les $[\text { anti- } \mathrm{N}]_{\mathrm{N}}$ antonymiques sélectionnent leurs bases différemment. Par exemple, il semble y avoir une plus forte proportion de $\mathrm{Nb}$ morphologiquement apparentés à des verbes parmi les $[n o n-\mathrm{N}]_{\mathrm{N}}$ contraires que parmi les $[a n t i-\mathrm{N}]_{\mathrm{N}}$ antonymiques, et une plus forte proportion de $\mathrm{Nb}$ morphologiquement apparentés à des noms parmi les $[\text { anti-N }]_{\mathrm{N}}$ antonymiques que parmi les $[n o n-\mathrm{N}]_{\mathrm{N}}$ contraires. D'autre part, et c'est en partie un corrélat de la répartition différente des types morphologiques, les $[n o n-\mathrm{N}]_{\mathrm{N}}$ contraires et les $[\text { anti- } \mathrm{N}]_{\mathrm{N}}$ antonymiques semblent faire porter des contraintes différentes sur le type sémantique de leur base. Mes données ont donc été annotées manuellement selon deux critères : (i) la complexité morphologique du nom base, (ii) la sémantique du nom base.

Premièrement, j'ai annoté les $[\text { non- } \mathrm{N}]_{\mathrm{N}}$ contraires et les $[\text { anti- } \mathrm{N}]_{\mathrm{N}}$ antonymiques en fonction de la complexité morphologique de leur nom base, en m'aidant de leur suffixe et, pour les cas difficiles, des indications fournies par le $T L F i$ à la rubrique étymologique de l'entrée du nom en question. J'ai ainsi distingué les noms déverbaux (suffixes -ade, -age, -ance/-ence, -ée, -ment, -ion, -ure); les noms désadjectivaux (suffixes -ité, -eur, -esse, -ise, -ice, -ion, -erie, -itude, -ancel-ence) ; les noms dénominaux (suffixes -ade, -age, -ance, -aille, -at, -ier, -ure); les lexèmes apparentés à des participes passés ou présents (PENSEE, VUE; EXISTANT) ; les lexèmes formés par composition et les unités polylexématiques (GRAND-MERE, GUERRE D'ALGERIE, ROMAN POLICIER, DEMANDE EN MARIAGE) ; les noms propres ; les lexèmes simples ou non construits en synchronie (ART, CHOSE, ESPACE). Le choix a été fait de ne pas attribuer de catégorie morphologique aux lexèmes portant les suffixes -isme ou -iste, étant donné la difficulté qu'il y a, dans ces cas, à décider du sens de la dérivation (Corbin, 1988; Roché, 2011).

Deuxièmement, chaque nom base a été classé selon son type sémantique. J'ai distingué les six classes sémantiques suivantes :

(i) Les artefacts, regroupant les artefacts non intellectuels (chaussure, maison), les artefacts informationnels et/ou artistiques (journal, article, livre, film, roman) ${ }^{22}$;

(ii) Les espèces naturelles, c'est-à-dire les animaux, végétaux, minéraux (oiseau, betterave, montagne) ;

(iii) Les êtres humains, qu'il s'agisse des êtres humains vus sous l'angle de leur activité professionnelle, de leur rôle social, de leur activité non professionnelle, ou encore de leur appartenance ethnique/nationale ou politique, religieuse ou intellectuelle (journaliste, héros, colombophile, Italien, communiste) ;

(iv) Les événements, classe qui rassemble à la fois les événements dotés d'étendue temporelle (guerre) et les événements ponctuels (apparition);

(v) Les abstractions, regroupant les objets abstraits (profit, valeur), les concepts propres à un domaine scientifique (l'être en philosophie), les qualités, sentiments et états (intelligence, tristesse, amour) ${ }^{23}$, et les comportements, qui désignent des qualités, facultés ou sentiments considérés sous un aspect attitudinal (générosité) ;

(vi) Les noms propres (dorénavant Npr), qu'ils désignent des êtres humains (Freud, de Gaulle), des événements (11 septembre 2001, guerre d'Algérie), des institutions (Sorbonne), ainsi que les toponymes (Japon, Palestine) ont été classés dans un groupe à part, étant donné qu'ils dénotent d'une manière qui diffère de celle des noms communs (cf. §4.1.2). 


\section{Examen et analyse des données}

\subsection{Examen des types de bases ${ }^{24}$}

\subsubsection{Mode de formation des $\mathrm{Nb}$}

On trouve dans le corpus de travail une majorité de bases simples à la fois parmi les $[\text { non-N }]_{\mathrm{N}}$ contraires et les $[\text { anti-N }]_{\mathrm{N}}$ antonymiques. Cette préférence est toutefois plus marquée dans le cas des $[\text { non-N }]_{\mathrm{N}}$ que dans celui des $[\text { anti-N }]_{\mathrm{N}}(70 \%$ contre $49 \%)$ :

(20) peut-être le pigeon des villes bénéficie-t-il d'un régime spécial, étant considéré comme non sauvage et même dégénéré par nos chers ornithologues? Un non oiseau, un OVNI : Oiseau Volant Nouvellement Interdit. (www)

(21) Bordeaux, Toulouse et Rennes sont, dans cet ordre, les équipes que vous jugez les plus décevantes dans ce trio d'outsiders en panne de résultats. Vos réactions à notre sondage. (...) Dans l'immédiat, vous placez les Girondins sur la première marche de notre antipodium (42\%), suivis de Toulouse $(36 \%)$ et de Rennes $(22 \%)$ que vous êtes assez nombreux à « sauver ». (Www)

Les $[\text { non- } \mathrm{N}]_{\mathrm{N}}$ contraires et les $[\text { anti-N }]_{\mathrm{N}}$ antonymiques diffèrent sur trois autres points. Tout d'abord, les bases déverbales sont en plus grande proportion parmi les $[n o n-\mathrm{N}]_{\mathrm{N}}$ contraires que parmi les [anti$\mathrm{N}]_{\mathrm{N}}$ antonymiques $(13 \%$ contre moins de $4 \%)$ :

(22) Vraiment pénible cette non-argumentation qui consiste à trouver pire pour justifier l'injustifiable. (www)

(23) Son aveu si caractéristique à Lady Melbourne, qu'il n'avance jamais que lorsque la femme a fait spontanément plus de la moitié du chemin, pourrait être la devise de cet anti-séducteur qui ne fait qu'un avec le séducteur malgré lui. (F)

Ces bases déverbales peuvent dénoter des objets sémantiquement divers (ici un événement (24) et un être humain (25)) ; j’y reviens au paragraphe suivant.

Deuxièmement, les bases dénominales se recontrent plus fréquemment parmi les $[\text { anti-N }]_{\mathrm{N}}$ antonymiques $(10 \%)$ que parmi les $[\text { non- } \mathrm{N}]_{\mathrm{N}}$ contraires (moins de $\left.4 \%\right)$ :

(24) Je crois que je suis un guerrier, et toi, que tu le veuilles ou non, tu es un anti-guerrier. (F)

(25) Le bulldozer dessine un non-paysage. Entre les colonies, le long des autoroutes de contournement, autour des check points, rien ne doit subsister qui puisse empêcher la visibilité. (F)

Enfin, les $[\text { anti-N }]_{\mathrm{N}}$ antonymiques fonctionnent très bien avec les noms propres, qui constituent un quart des bases $(25 \%)$, ce qui n'est pas le cas des $[\text { non- } \mathrm{N}]_{\mathrm{N}}$ contraires $(10 \%$ seulement $)$. Ce point est développé au paragraphe suivant $(\$ 4.1 .2)$, consacré à la sémantique des noms bases.

Le tableau 1 récapitule la distribution des types morphologiques des noms bases des $[\text { non-N }]_{\mathrm{N}}$ contraires et des $[\text { anti-N }]_{\mathrm{N}}$ antonymiques.

\begin{tabular}{|l|r|r|r|r|}
\hline Type de base & non- & Ratio/total & anti- & Ratio/total \\
\hline simplex & 89 & $\mathbf{7 0 , 0 8 \%}$ & 82 & $\mathbf{4 9 , 4 0 \%}$ \\
nom déverbal & 17 & $\mathbf{1 3 , 3 9 \%}$ & 6 & $3,61 \%$ \\
nom désadjectival & 3 & $2,36 \%$ & 10 & $6,02 \%$ \\
nom dénominal & 5 & $3,94 \%$ & 17 & $\mathbf{1 0 , 2 4 \%}$ \\
participe (passé, présent) & 3 & $2,36 \%$ & 0 & $0,00 \%$ \\
dérivé en -iste ou -isme & 0 & $0,00 \%$ & 3 & $1,81 \%$
\end{tabular}




\begin{tabular}{|l|r|r|r|r|} 
unité polylexématique & 8 & $6,29 \%$ & 6 & $3,61 \%$ \\
nom propre & 2 & $1,57 \%$ & 42 & $\mathbf{2 5 , 3 0 \%}$ \\
\hline TOTAL & $\mathbf{1 2 7}$ & $\mathbf{1 0 0 , 0 0 \%}$ & $\mathbf{1 6 6}$ & $\mathbf{1 0 0 , 0 0 \%}$ \\
\hline
\end{tabular}

Tableau 1. Répartition des bases selon leur mode de construction morphologique.

\subsubsection{Sémantique des $\mathrm{Nb}$}

Les $[n o n-\mathrm{N}]_{\mathrm{N}}$ contraires ont une préférence pour les bases dénotant des artefacts $(37 \%)$ :

(26) J'ai été tenté de taper sur les marinières, mais j'ai trouvé pire avec les tennis en toile, style Victoria ou Bensimon. La non-chaussure par excellence, qui m'évoque irrémédiablement les mamies condamnées par leurs cors au port de chaussures confortables. (www)

Certains d'entre eux sont des artefacts intellectuels, c'est-à-dire qu'ils peuvent dénoter soit des objets physiques, soit des objets non physiques (plus précisément, des idéalités, dont le mode d'être implique une dimension temporelle, cf. Flaux \& Van de Velde, 2000). Dans le cas de la lecture contraire, la préfixation par non- (l'effet est le même avec la préfixation par anti-) vise la facette non physique du référent :

(27) Il avait considéré Casque d'or comme un non film, l'avait scientifiquement démontré comment ç'aurait pu devenir un film si lui, Clouzot, l'avait mis en scène à la place de Jacques. (F)

Les $[\text { non- } \mathrm{N}]_{\mathrm{N}}$ contraires sélectionnent également un grand nombre de bases dénotant des événements $(28 \%)$ :

(28) C'est le grand paradoxe de cette élection à la présidence du Medef. Jamais la situation de la France n'a été aussi critique (...) et personne, dans cette non-campagne, ne parle des entreprises, des entrepreneurs. Non, on ne parle que des statuts de l'organisation patronale, de la tentative de sa présidente sortante de faire un 3ème mandat (...) (www)

La différence avec les $[a n t i-\mathrm{N}]_{\mathrm{N}}$ antonymiques est ici flagrante : seuls $4 \%$ d'entre eux sont construits sur des bases dénotant des événements :

(29) Il va jusqu'à la chaise où sont posés ses vêtements, il commence à se rhabiller. (...) premier acte : le caleçon, acte deux: la liquette, acte trois : les chaussettes avant le pantalon, enfin acte quatre : la cravate et le geste rond qu'elle implique - cet anti strip-tease qui ne cesse d'être ridicule que s'il s'accomplit dans la bonne humeur, Laurent, parce qu'il est sombre, en fait une espèce de drame. $(\mathrm{F})$

L'autre grande différence concerne les bases dénotant des noms propres : elles constituent $25 \%$ des [anti$\mathrm{N}]_{\mathrm{N}}$ antonymiques contre seulement $1,6 \%$ des $[\text { non-N }]_{\mathrm{N}}$ contraires. Dans le cas des $[\text { anti- } \mathrm{N}]_{\mathrm{N}}$, il s'agit principalement de noms propres d'êtres humains ( 28 sur $38 \mathrm{Npr}$ ) :

(30) François Hollande est l'anti-de Gaulle par excellence. Dans les crises, il ne renverse pas la table. Il gagne du temps. Toute sa vie, il s'est méfié du lyrisme, des grands mots, des grands hommes et des révolutions. (www)

Mais on trouve aussi des toponymes (31) et des Npr dénotant des institutions (32) :

(31) Plus d'un trait découle de cette opposition qui fait du Canada un anti-Japon. Le Japonais ne craint ni le vent, ni le froid. Dans sa maison de papier, tout à fait impropre au chauffage, le vent entre et sort comme chez lui. (F)

(32) Après 1968, Paris IV servit de refuge à la fraction la plus conservatrice du monde académique, tandis que Paris VIII, qui se voulait une sorte d'anti-Sorbonne, concentrera l'avant-garde intellectuelle et politique de l'époque. (www) 
Les Npr qui servent de base à la préfixation en non- et en anti- dans les lectures respectivement contraire et antonymique sont dans un emploi métaphorique. Je reprends ici Kleiber (1981) qui utilise le terme de métaphore pour désigner les exemples du type Paul est un vrai Harpagon (Kleiber, 1981 : 358), mais tous les auteurs ne s'accordent pas sur ce point, par exemple Flaux (1991). En théorie, un NPr est non descriptif, il ne renvoie à aucune propriété du particulier qu'il désigne. Dans sa fonction référentielle, habituelle, il « désigne un particulier sans le décrire, ni le classifier, mais en vertu d'une convention ad hoc de dénomination qui associe directement et avec un lien durable la forme phonique ou graphique du NPr au particulier visé » (Jonasson, $1994: 65$ ) : c'est un désignateur rigide (Kripke, 1972) ou direct (Kleiber, 1981). Mais dans les exemples décrits dans cet article, il est fait un usage prédicatif du dérivé $[\text { anti-N }]_{\mathrm{N}}$, et cette interprétation est possible justement parce que les NPr permettent l'usage prédicatif. Ce qui est visé, ce sont des propriétés stéréotypiques de certains individus porteurs de ces NPr «culturellement saillants » (Jonasson, 1994) ${ }^{25}$. Il s'agit dans chaque cas de personnes qui font partie de l'univers de connaissance des scripteurs qui produisent ces énoncés. Bien évidemment, les scripteurs supposent que leurs lecteurs disposent également de cet ensemble de connaissances sociales qui fait que de Gaulle (30) est identifié comme un Président de la République française théâtral et charismatique, Freud (2) comme le père de la psychanalyse, méthode complexe d'investigation de la psyché et auteur d'une œuvre dense. Dans le cas de NPr de lieux (31) ou d'institutions (32), le mécanisme reste le même : par exemple, au NPr Sorbonne est attaché un certain nombre de propriétés stéréotypiques ; la première partie de l'énoncé en donne d'ailleurs un aperçu («fraction la plus conservatrice du monde académique $\gg)$.

Le corpus contient $66[\text { anti- } \mathrm{N}]_{\mathrm{N}}$ antonymiques avec une base dénotant un humain, dont 28 noms propres, contre $19[\text { non- } \mathrm{N}]_{\mathrm{N}}$ contraires dénotant un humain, et parmi ceux-ci, aucun nom propre : lorsqu'il s'agit de mettre en relief certains traits de l'humain porteur du nom propre base, et d'en prédiquer l'absence chez le référent du nom dérivé, les locuteurs préfèreront semble-t-il former un dérivé en anti- qu'un dérivé en $n o n-{ }^{26}$. Les deux bases noms propres rencontrés dans le corpus de $[\text { non-N }]_{\mathrm{N}}$ contraires réfèrent pour l'un à un événement (GUERRE D'ALGERIE), pour l'autre à un toponyme (PALESTINE).

Enfin, les bases dénotant des êtres humains sont plus nombreuses dans le cas des $[a n t i-\mathrm{N}]_{\mathrm{N}}$ antonymiques $(23 \%)$ que dans celui des $[\text { non- } \mathrm{N}]_{\mathrm{N}}$ contraires $(15 \%)$. En (33), une relation métonymique relie l'artefact blouson noir à un être humain :

(33) L'accusé, tel que vous me le décrivez, est une sorte d'anti-blouson noir, le brave type que rien ne prédisposait au meurtre et qui y a été conduit malgré lui. (F)

(34) Jean-Pierre Pernod est un non journaliste, dans un non journal, ou le degré zéro de l'information fait pour les plus de 85 ans plus très frais psychiquement. (www)

Enfin, on rencontre parmi les $[a n t i-\mathrm{N}]_{\mathrm{N}}$ antonymiques une proportion égale $(23 \%)$ de bases dénotant des abstractions (35) et des artefacts (36) :

(35) La Chine venait donc prendre la place d'une anti-origine : la plus profonde, la plus ancestrale, la race des ancêtres aux yeux bridés, mais aussi la plus invraisemblable, donc la plus indolore, impersonnelle, sans couleurs enfantines, juste un puzzle de mirages. (F)

(36) Moi qui souffre, comme déjà dit, d'hyperacousie, je regrette de très mal supporter cette sorte d'anti-sonotone, qui ajoute au bruit du dehors un bourdonnement intérieur bien incapable de le couvrir. (F)

Comme dans le cas des $[\text { non-N }]_{\mathrm{N}}$ contraires, les artefacts sont surtout des objets fabriqués informationnels ou, comme en (37), artistiques :

Nous sommes donc face à une dérive du roman policier, et nous entrons dans le monde du roman d'un justicier. Pour autant, très vite les circonstances vont faire qu'il s'agit en fait d'un anti-roman policier. (www)

Le tableau 2 récapitule la distribution des types sémantiques des noms bases des $[\text { non- } \mathrm{N}]_{\mathrm{N}}$ contraires et des $[\text { anti-N }]_{\mathrm{N}}$ antonymiques. 


\begin{tabular}{|l|r|r|r|r|}
\hline Type de base & non- & Ratio/total & anti- & Ratio/total \\
\hline artefact & 47 & $\mathbf{3 7 , 0 1 \%}$ & 38 & $\mathbf{2 2 , 8 9 \%}$ \\
espèce naturelle & 9 & $7,09 \%$ & 3 & $1,81 \%$ \\
être humain & 19 & $14,96 \%$ & 38 & $\mathbf{2 2 . 8 9 \%}$ \\
événement & 33 & $\mathbf{2 7 , 9 8 \%}$ & 7 & $4,22 \%$ \\
abstraction & 17 & $13,39 \%$ & 38 & $\mathbf{2 2 , 8 9 \%}$ \\
nom propre & 2 & $1,57 \%$ & 42 & $\mathbf{2 5 , 3 0 \%}$ \\
\hline TOTAL & $\mathbf{1 2 7}$ & $\mathbf{1 0 0 , 0 0 \%}$ & $\mathbf{1 6 6}$ & $\mathbf{1 0 0 , 0 0 \%}$ \\
\hline
\end{tabular}

Tableau 2. Répartition des bases selon leur classe sémantique.

Enfin, si l'on examine les dérivés du TLFi et ceux de Frantext, seuls 12 d'entre eux se retrouvent à la fois dans un $[n o n-\mathrm{N}]_{\mathrm{N}}$ contraire et dans un $[\text { anti }-\mathrm{N}]_{\mathrm{N}}$ antonymique : il s'agit de ART, ARTISTE, CHOSE, COMMUNICATION, HEROS, HISTOIRE, LANGAGE, LIVRE, MAISON, MONDE, MUSIQUE, POESIE. Ceci suggère que, dans ce type de dérivés, les préfixations en non- et en anti- ne sont pas équivalentes.

\subsection{Interprétation des dérivés}

\subsubsection{Interprétation des $[\text { non- } \mathrm{N}]_{\mathrm{N}}$ contraires}

Dans les [non- $\mathrm{N}]_{\mathrm{N}}$ contraires, il ne fait pas de doute que le référent du dérivé appartient à la catégorie du référent du $\mathrm{Nb}$, mais le locuteur refuse cette catégorisation. Par exemple, en (38), un non-musée est un musée - mais le locuteur ne le reconnaît pas comme tel :

(38) Pour les excursions sur Conakry, rien à voir là-bas. Tout ce qui méritait d'être vu a été détruit. Le musée Sandervalia est un non-musée mais il y a toujours des coins sympas pour boire une bière et manger un bon plat surtout en banlieue. (www)

Dans ces dérivés, non- exprime fondamentalement un sens de négation qui mêle deux caractéristiques : (i) un sens axiologique, qui correspond à une évaluation négative : un $[\text { non-N }]_{\mathrm{N}}$ n'est pas un bon $\mathrm{Nb}$; (ii) un emploi métalinguistique, qui correspond à un jugement porté par le locuteur sur la dénomination du $\mathrm{N}$ dérivé, sur l'assertabilité de " $\mathrm{N}$ est un $\mathrm{Nb}$ » : un $[\text { non- } \mathrm{N}]_{\mathrm{N}}$ " n'est pas digne de s'appeler un $\mathrm{Nb}$ », « est tellement mauvais qu'il ne mérite pas le nom de $\mathrm{Nb}$ ». Un non-musée (38) est si inintéressant qu'il ne mérite pas le nom de musée (et ne mérite pas d'être vu, si l'on en croit la glose qui accompagne NONMUSEE), tout comme une non-chaussure (26) est inélégante au point de ne pas mériter le nom de chaussure. Pour donner un dernier exemple, en (39), les réfugiés hébergés à Sangatte ne sont pas considérés comme des personnes à part entière :

(39) Sangatte : un non-lieu, pour des non-gens. (...) Inauguré en septembre 1999 le « centre d'hébergement et d'accueil d'urgence humanitaire » de Sangatte (...), a accueilli 78000 étrangers depuis son ouverture. Avant c'est dans la rue qu'ils dormaient en attendant chaque nuit de gagner l'Angleterre. Fin août, ces indésirables étaient environ 1700 (un record). Le 16 octobre dernier ils étaient 1085. Mais comment être sûr de ces chiffres puisqu'à part la Croix-Rouge qui « gère » ce centre et quelques agités, tout le monde fait semblant qu'ils n'existent pas... (www)

Étant donnée cette valeur évaluative et métalinguistique, les $[\text { non-N }]_{\mathrm{N}}$ contraires ne sont pas classifiants, mais qualifiants. Contrairement à ce qui est suggéré par Algeo (1971) et par Horn (2002a, 2002b), ils ne se caractérisent pas par une relation d'hypo/hyperonymie: un non-musée n'est pas un type ${ }^{27}$ de musée, une non-chaussure n'est pas un type de chaussure, des non-gens ne sont pas des types de gens.

De plus, ces dérivés n'ont pas pour fonction d'attribuer une dénomination à une réalité extralinguistique. On parle de dénomination lorsqu'il y a un « lien référentiel constant » entre le signe et un objet du monde extralinguistique : l'association référentielle «n'a pas pour but une désignation uniquement momentanée, transitoire et contingente, de la chose, mais au contraire l'établissement d'une règle de fixation 
référentielle qui permet l'utilisation ultérieure du nom pour l'objet dénommé » (Kleiber, 1984:80). Ces dérivés doivent être vus comme une manifestation de la créativité lexicale des locuteurs, c'est pourquoi ils sont rarement attestés dans les dictionnaires : la nomenclature du TLFi n'enregistre que 11 [non-N] $]_{\mathrm{N}}$ contraires, contre $58[\text { anti- } \mathrm{N}]_{\mathrm{N}}$ antonymiques. Enfin, il est très fréquent que le locuteur éprouve le besoin de préciser le sens qu'il donne au $[n o n-\mathrm{N}]_{\mathrm{N}}$, en mentionnant dans le contexte immédiat les propriétés stéréotypiques du nom sur lequel porte non-. Il n'y a pas nécessairement d'accord entre les locuteurs sur les traits considérés comme stéréotypiques, par exemple dans le cas de NON-FEMME en (40) :

a. Bon, mon problème, c'est que je n'ai pas envie de devenir ce modèle de féminité exacerbée. Je suis petite, menue, je ne me maquille pas, ne porte pas de jupe ni de talons, j'ai les cheveux et les ongles courts. Ah oui ! Et je n'ai pas de poitrine ! (...) Mais est-ce que ça fait de moi une non-femme ?? (www)

b. Je suis dingue de plantations. Les fleurs, par contre, bof, je m'en fiche ! Serais-je une nonfemme? (www)

Je reprends ici la distinction faite par Anscombre (2001) entre stéréotypes primaires et stéréotypes secondaires. Les premiers sont attachés de façon stable à un terme dans une communauté linguistique donnée ; les seconds sont attachés localement à l'occurrence d'un terme, et dépendent plus fortement du contexte. Avec les stéréotypiques secondaires, «le locuteur émet une opinion qui lui est propre, sans cependant nécessairement se démarquer de la communauté linguistique à laquelle il se présente comme appartenant » (Anscombre, $2001: 64)$. Étant donné le rôle du contexte dans l'interprétation des $[\text { non-N }]_{\mathrm{N}}$ contraires, il semble que ces dérivés mettent principalement en jeu des stéréotypes secondaires.

\subsubsection{Interprétation des $[a n t i-\mathrm{N}]_{\mathrm{N}}$ antonymiques}

On retrouve dans les $[\text { anti-N }]_{\mathrm{N}}$ antonymiques le sens originel du préfixe grec àv $\tau_{i}$ : l'idée de face-à-face « en miroir », comme le souligne Hathout (2009) - et d'opposition (cf. §2.3.2). Premièrement, le sens du dérivé est relativement neutre : il y a inversion de propriétés stéréotypiques, mais sans jugement de valeur du locuteur sur l'appartenance du référent du dérivé à la catégorie dénotée par le $\mathrm{Nb}$ :

(41) La preuve que le rock n'était pas mort avec Buddy Holly, mais aussi que chaque musique crée un anti-monde. Une bulle irisée, où se déplace l'auditeur, plus grand, plus fort et plus libre et plus beau que sur terre. (F)

Deuxièmement, ces dérivés ont souvent un sens teinté d'adversativité (cf. les dérivés du type ANTILIMACES $_{\mathrm{A} / \mathrm{N}}$ ), comme l'illustrent semble-t-il ANTI-JAPON en (31), ANTI-SORBONNE en (32), mais aussi ANTI-TECHNIQUE en (42) :

(42) le fondateur de la cybernétique, Norbert Wiener, (...) fournit lui-même aux croisés de l'antitechnique des arguments qui ne sont pas négligeables, lorsqu'il se risque à prophétiser : « La révolution industrielle moderne est fatalement conduite à dévaloriser le cerveau humain à tout le moindre dans ses décisions les plus simples et les plus routinières ». $(\mathrm{F})$

Concernant le statut de ces dérivés, on ne peut pas, contrairement à ce qui est affirmé notamment par Heyna (2008, 2009, 2013) et Hathout (2009), parler de relation d'hypo/hyperonymie. Le trait "vrai», invoqué pour caractériser la différence entre les co-hyponymes (entre, par exemple, un « vrai » guerrier et un guerrier non prototypique (24)), n'est pas un trait de nature classifiante, mais qualifiante. Un antiroman policier (37) n'est pas une sorte de roman policier, tout comme une anti-voiture n'est pas une sorte de voiture $(2 \mathrm{~b})$.

Par ailleurs, on peut se demander s'il faut attribuer le statut de dénomination aux $[\text { anti-N }]_{\mathrm{N}}$ antonymiques. On remarque que les $[\text { anti-N }]_{\mathrm{N}}$ antonymiques sont facilement employés avec des enclosures (p. ex. sorte de (2a), (8), (32), (33), (36), par excellence (26), (30) mais aussi genre de) - or ces enclosures ne peuvent porter que sur des référents déjà dotés d'une catégorie sémantique, puisqu'elles opèrent une recatégorisation (cf. §2.2). À titre indicatif, une rapide interrogation du moteur de recherche Google ${ }^{T M}$ (pages : France) faite le 11/12/2013 ramène 1030000 résultats pour « une sorte d'anti » contre 66000 
pour « une sorte de non », et 937000 résultats pour « un genre d'anti» et seulement 52400 pour « un genre de non». Ceci suggère que les $[a n t i-\mathrm{N}]_{\mathrm{N}}$ antonymiques sont davantage des dénominations. Néanmoins, le contexte joue un rôle important dans l'identification des propriétés affectées par la préfixation, ce qu'illustrent les occurrences d'ANTI-WESTERN en (43) :

a. Un roman sur le mal et un regard sans concession, qui exhibe la face cachée de l'histoire américaine, scandée par les massacres, les exactions, les larcins etc. En somme un anti western. Les décors sont les mêmes que dans les westerns hollywoodiens, mais la conquête n'aboutit à rien, sinon au vide, à la vacuité de l'immensité désertique où la violence se déploie en pure perte. (www)

b. Je conseille Blessés (Actes Sud), un roman contemporain de l'Américain Percival Everett, paru en France il y a quinze jours. Il s'agit d'un anti-western où le héros est un cow-boy noir. Le livre aborde des thèmes comme le racisme et l'exclusion dans les États-Unis d'aujourd'hui. Il y est aussi question d'homosexualité. C'est un roman très touchant, où l'on retrouve un peu l'atmosphère des grands espaces américains des livres de Jim Harrison. (www)

\section{Conclusions et perspectives}

Ce travail a montré que les $[\text { non- } \mathrm{N}]_{\mathrm{N}}$ contraires et les $[\text { anti-N }]_{\mathrm{N}}$ antonymiques ont en commun de sélectionner les propriétés stéréotypiques que le locuteur attache au référent du nom base. L'examen des données a toutefois révélé que ces deux lectures ne recouvrent pas des réalités identiques. Premièrement, les préfixations en non-contraire et anti- antonymique ne sélectionnent pas les mêmes types de bases, ce qui suggère qu'elles ne sont pas sensibles aux mêmes propriétés. Deuxièmement, les $[n o n-\mathrm{N}]_{\mathrm{N}}$ contraires ont une valeur axiologique et non- a dans ces dérivés un emploi métalinguistique, tandis que les [anti$\mathrm{N}]_{\mathrm{N}}$ antonymiques sont plus neutres et anti- y exprime essentiellement l'opposition. Par ailleurs, le rapport qu'entretiennent les $[n o n-\mathrm{N}]_{\mathrm{N}}$ contraires et les $[\text { anti- } \mathrm{N}]_{\mathrm{N}}$ antonymiques avec leur nom base n'est pas de l'ordre de l'hypo/hyperonymie. Enfin, les $[\text { non-N }]_{\mathrm{N}}$ contraires ne sont pas des dénominations ; des recherches plus approfondies doivent être menées afin de déterminer s'il faut tirer une conclusion similaire pour les [anti-N $]_{\mathrm{N}}$ antonymiques, qui semblent toutefois beaucoup plus « dénominatifs » que les $[\text { non }-\mathrm{N}]_{\mathrm{N}}$ contraires.

À partir de ces constats, diverses pistes de travail peuvent être envisagées. Le cas des noms propres fonctionnant comme input à la préfixation (ANTI-FREUD (2a), ANTI-DE GAULLE (30)), par exemple, mérite un examen approfondi. Les noms propres sont des dénominations bien particulières; a priori non descriptifs, ils doivent être «métaphorisés » (Kleiber, 1981) pour renvoyer aux propriétés des individus qu'ils désignent. Le fait que ces propriétés ne soient pas directement accessibles explique peut-être la faible appétence de non- pour ce type de bases.

Concernant le lien sémantique particulier qui unit le dérivé à son $\mathrm{Nb}$ dans les $[\text { non-N }]_{\mathrm{N}}$ contraires, un rapprochement pourrait éventuellement être tenté avec les troponymes tels que théorisés par Fellbaum $(1990,1999)$ pour le domaine verbal: une relation troponymique est une relation hiérarchique d'implication lexicale entre deux verbes, qui peut être exprimée par la formule «faire V1, c'est faire V2 d'une façon particulière ", par exemple rouler / bouger, sautiller / sauter (pour ce dernier cas de figure, cf. Stosic \& Amiot, 2011). La notion de manière est fondamentale dans la troponymie. Fellbaum parle d'implication lexicale pour décrire cette relation unilatérale entre deux verbes, c'est-à-dire que dans un rapport de troponymie, la phrase « quelqu'un V1 » implique logiquement la phrase «quelqu'un V2 ». À cela s'ajoute l'idée de hiérarchie : les verbes en bas de la hiérarchie ont plus de composants sémantiques que les verbes de base (par exemple rouler par rapport à bouger, sautiller par rapport à sauter), mais il ne s'agit pas d'une hiérarchie du type hypo/hyperonymie. Mais il reste à voir si cette approche est transférable au domaine nominal.

Enfin, alors que le sémantisme d'anti- pourrait être rapproché de celui du préfixe contre-, il serait intéressant de comparer non- avec les préfixes évaluatifs (p. ex. pseudo-, semi-, sous-) (44) : 
(44) a. La littérature historique renferme beaucoup de fausse histoire, de pseudo-histoire, de nonhistoire. $(T L F i)$

b. les hormones femelles du Dr Frank m'ont rendu ma virilité, des poils noirs ont même poussé sur ma poitrine imberbe, c'est comme en août 44, j'ai cessé d'être ein Untermensch, un soushomme, voire un Unmensch, un non-homme, quand je suis sorti pour la première fois de ma cachette, enterré depuis d'interminables mois. (F)

On constate une fois de plus que, dans ces dérivés, non- n'exprime pas une opposition, mais un jugement appréciatif porté par le locuteur sur un défaut de propriétés qui va de pair avec un écart à une norme. À n'en pas douter, non- et anti- évoluent dans deux arènes différentes.

\section{Références bibliographiques}

Algeo, J. (1971). The voguish uses of Non. American Speech, vol. 46, $n^{\circ}$ 1/2, 87-105.

Anscombre, J.C. (2001). Dénomination, sens et référence dans une théorie des stéréotypes nominaux. Cahiers de proxématique (36), 43-72

Anscombre, J.C. (1994). L'insoutenable légèreté morphologique du préfixe négatif in- dans la formation d'adjectifs. Linx. Revue des linguistes de l'université Paris X Nanterre, (5), 299-321.

Aristote. (2008). Métaphysique. Paris : Garnier Flammarion. Traduction M.P. Duminil et A. Jaulin.

Bauer, L. (1983). English word-formation. Cambridge: Cambridge University Press.

Corbin, D. (1988). Une hypothèse à propos des suffixes-isme,-ique,-iste du français : la troncation réciproque. Aspects de linguistique française, Hommage à QIM Mok, 63-75.

Corbin D. (1980). Contradictions et inadéquations de l'analyse parasynthétique en morphologie dérivationnelle. In Théories linguistiques et traditions grammaticales, Dessaux-Berthoneau A.-M. (ed.), Lille : Presses universitaires de Lille, 181-224.

Cruse, A. (1986). Lexical semantics. Cambridge: Cambridge University Press.

Dal, G., Grabar, N., Lignon, S., Plancq, C., Tribout, D., Yvon, F. (2007). Les adjectifs de forme in-X-able en français. La négation dans les langues romanes, 205-224.

Di Sciullo, A.M. et Tremblay, M. (1993). Négation et interfaces. Toronto Working Papers in Linguistics 12, $n^{\circ} 1,75-$ 89.

Dugas, E. (2013). [non(-)Adj] sequences in contemporary French: morphological negation, syntactic negation, or in between?. Communication faite lors du Symposium "Morphology and its interfaces", Université Lille 3, 12-13 Septembre 2013.

Dugas, E. (2012). La négation en morphologie : le cas des formes nominales en non- en français, mémoire de Master 2 (non publié), Université Lille 3.

Durand, J. (1982). À propos du préfixe anti- et de la parasynthèse en français. Occasional Papers from the Language Center, University of Essex, vol. 25, 1-35.

Fellbaum, C. (1999). La représentation des verbes dans le réseau sémantique WordNet. Langages, vol. 33, $n^{\circ} 136$, $27-40$.

Fellbaum, C. (1990). English verbs as a semantic net. International Journal of Lexicography 3, $n^{\circ} 4,278-301$.

Flaux, N. (1991). L'antonomase du nom propre ou la mémoire du référent. Langue française, $n^{\circ}$ 92, 26-45.

Flaux, N., Van de Velde, D. (2000). Les noms en français : esquisse de classement. Paris : Ophrys.

Fradin, B. (1997a). Esquisse d'une sémantique de la préfixation en anti- . Recherches linguistiques de Vincennes, $n^{\circ}$ 26, 87-112.

Fradin, B. (1997b). Une préfixation complexe : le cas de anti-. Neuphilologische Mitteilungen, vol. 98, $n^{\circ}$ 4, 333-349. 
Fradin, B. (1984). Langue, discours, lexique. Linx 10, Centre de recherche de l'Université de Paris X Nanterre, 159165.

Gaatone, D. (1971). Étude descriptive du système de la négation en français contemporain. Genève : Librairie Droz.

Geeraerts, D. (2008). Prototypes, stereotypes, and semantic norms. In Kristiansen G., R. Dirven (eds.), Cognitive Sociolinguistics: Language Variation, Cultural Models, Social Systems, Berlin/New York: Mouton de Gruyter, 21-44.

Geeraerts, D. (2006). Prospects and problems of prototype theory. In Geeraerts, D. (ed.), Cognitive Linguistics: Basic Readings, Berlin/New York: Mouton de Gruyter, 141-165.

Hathout, N. (2009). Contributions à la description de la structure morphologique du lexique et à l'approche extensive en morphologie. Habilitation à diriger des recherches. Universités Toulouse II-Le Mirail.

Hathout, N., Levkovych, O., Plénat, M. (2004). Morphologie et recyclage : le cas de anti-. Communication aux $3^{\mathrm{e}}$ Décembrettes, Toulouse (non publié).

Heyna, F. (2013). Antirides et antihéros : valeurs adversative et antonymique des dérivés en anti-. In Larrivée P. (ed.) La linguistique de la contradiction, Bruxelles : Peter Lang, 53-72.

Heyna, F. (2009). The Use of Anti- in Contemporary French: A Case of Degrammaticalization? In Rossari, C., C. Ricci \& A. Spiridon (eds.), Grammaticalization and pragmatics: facts, approaches, theoretical issues 5, 193-219.

Heyna, F. (2008). Sémantisme et potentiel argumentatif des dérivés dénominaux en anti. Discours, vol. 2, URL : http://discours.revues.org/2022.

Horn, L.R. (2002a). Uncovering the un-word: a study in lexical pragmatics. Sophia linguistica, vol. 49, 1-64.

Horn, L. R. (2002b). Assertoric inertia and NPI licensing. Chicago Linguistic Society, vol. 38, $n^{\circ}$ 3, 55-82.

Horn, L. R. (1989). A Natural History of Negation. Chicago, IL: University of Chicago Press.

Huot, H. (2007). La préfixation négative en français moderne. In Floricic F. (ed.), La négation dans les langues romanes. Amsterdam : Benjamins, 177-204.

Jonasson, K. (1994). Le nom propre. Constructions et interprétations. Louvain-la-Neuve : Duculot.

Kalik, A. (1971). La caractérisation négative. Le Français Moderne, (2).

Katz, J. J. (1972). Semantic Theory. New York: Harper \& Row.

Kleiber, G. (1990). La sémantique du prototype. Paris : PUF.

Kleiber, G. (1984). Dénomination et relations dénominatives. Langages, (76), 77-94.

Kleiber, G. (1981). Problèmes de référence : descriptions définies et noms propres. Paris : Klincksieck.

Kleiber, G., Riegel, M. (1978). Les grammaires floues. In Martin R. (ed.), La recevabilité en linguistique, Paris : Klincksieck, 67-123.

Kripke, S. A. (1972). 'Naming and Necessity'. In Davidson D. \& Harman G. Semantics of Natural Language, Reideil, Dordrecht, 253-255.

Lakoff, G. (1973). Hedges: a Study in Meaning Criteria and the Logic of Fuzzy Concepts. In Papers from the $9^{\text {th }}$ Regional Meeting of the Chicago Linguistic Society, 183-228.

Lyons, J. (1977). Semantics. 2 vols. New York: Cambridge University Press.

Plag, I. (2003). Word-formation in English. Cambridge: Cambridge University Press.

Pottier (1965). La definition sémantique dans les dictionnaires. Travaux de linguistique et de littérature $3 n^{\circ} 1,33-39$.

Putnam, H. (1975). The meaning of meaning. In Gunderson K. (ed.), Language, Mind and Knowledge, Minneapolis : University of Minnesota Press, 131-193.

Rey, A. (1968). Un champ préfixal : les mots français en anti-. Cahiers de lexicologie, vol. 12, $n^{\circ}$ 1, 37-57.

Roché, M. 2011. Quel traitement unifié pour les dérivations en -isme et en -iste? In Roché M., G. Boyé, N. Hathout, S. Lignon \& M. Plénat (eds.). Des unités morphologiques au lexique, Paris : Hermès / Lavoisier, 69-143. 
Rosch, E. (1978). Principles of Categorization. In Rosch E. \& B. Llyod (eds.), Cognition and Categorization, Hillsdale : Laurence Erlbaum Ass., 27-48.

Rosch, E. \& Mervis, C. B. (1975). Family resemblances: Studies in the internal structure of categories. Cognitive psychology, 7(4), 573-605.

Schäfer, R. et Bildhauer, F. (2012). Building large corpora from the web using a new efficient tool chain. In N. Calzolari et al. (eds.), Proceedings of the Eight International Conference on Language Resources and Evaluation, Istanbul, ELRA, 486-493.

Stosic, D., \& Amiot, D. (2011). Quand la morphologie fait des manières : les verbes évaluatifs et l'expression de la manière en français. In Amiot D. et al. (eds.), Ars Grammatica. Hommages à Nelly Flaux, 403-430.

Wierzbicka, A. (1985). Lexicography and Conceptual Analysis. Ann Arbor, Karoma

Wittgenstein, L. (1953). Philosophical investigations. New York : The McMillan Co.

Zimmer, K.E. (1964). Affixal negation in English and other languages: an investigation of restricted productivity. Supplement to Word, vol. 20, $n^{\circ} 2$, Monograph $n^{\circ} 5$.

Zribi-Hertz, A. (1973). Recherches sur la préfixation productive en français moderne. Thèse de doctorat de $3^{\mathrm{e}}$ cycle, Université Paris 8 : Vincennes.

${ }^{1}$ Cette interprétation des $[a n t i-\mathrm{N}]_{\mathrm{N}}$ prend le nom d'《emplois antipodaux » chez Fradin (1997a, 1977b) et d'« interprétation logique » chez Hathout (2009).

${ }^{2}$ Rey (1968) parle de «valeur d'opposition », Corbin (1980) d' « antagonisme » et d' " opposition polémique », Fradin (1997a, 1997b) d' " emplois oppositifs ».

${ }^{3}$ http://atilf.atilf.fr/

${ }^{4}$ http://www.frantext.fr/

${ }^{5}$ « Two contrary terms represent areas at the opposite poles of a given dimension and leave some room for other possibilities between them ». (Toutes les traductions sont les miennes).

${ }^{6}$ « The essence of a pair of complementaries is that between them they exhaustively divide some conceptual domain into two mutually exclusive compartments, so that what does not fall into one of the compartments must necessarily fall into the other $»$.

${ }^{7}$ La notion de « ressemblance de famille » a été mobilisée pour la première fois par Wittgenstein (1953) pour décrire ce qui relie les différentes propriétés attachées à un référent ; cette relation prend la forme $\mathrm{AB}, \mathrm{BC}, \mathrm{CD}, \mathrm{DE}$ : « each item has at least one, and probably several, elements in common with one or more items, but no, or few, elements are common to all items » (Rosch \& Mervis, $1975: 574$ sq.).

8 «stereotypes are prototypes seen from a social angle ».

9 « a socially determined minimum set of data with regard to the extension of the category ».

${ }^{10}$ Toute communauté linguistique «possède au moins quelques termes dont les 'critères' sont connus seulement d'une partie des locuteurs qui acquièrent ces termes, et dont l'emploi par d'autres locuteurs dépend de la coopération structurée entre eux et les locuteurs des autres groupes » ( « every linguistic community exemplifies the sort of division of linguistic labor (...): that is, possesses at least some terms whose 'criteria' are known only to a subset of the speakers who acquire the terms, and whose use by the other speakers depends upon a structured cooperation between them and the speakers in the relevant subsets » (Putnam, $1975: 146$ ).

11 «possesses $(\ldots)$ the accidents, but not the substance named ».

12 «possessing the value, but not the surface characteristics or acknowledged identity of »; « the thing possesses the substance though not the accidents ».

13 « do not satisfy the structural criteria for the category $\mathrm{X}$ but share its function, evoking a superset category encompassing both $\mathrm{X}$ and $u n-\mathrm{X} »$. 
14 « is a member of the category (...), but a peripheral or non-prototype member (...), one lacking a functionally significant (but not criterial) property associated with the category in question ».

15 « words whose meaning implicitely involves fuzziness: words whose job is to make things fuzzier or less fuzzy ».

${ }^{16}$ Ce préfixe est homographe du préfixe anti- issu du préfixe latin ante-, que l'on retrouve notamment dans les dérivés ANTICHAMBRE, ANTIDATER. Hathout (2009) rassemble d'ailleurs ces deux préfixes autour du sens 'en face' du préfixe grec $\alpha \dot{v} \tau i$. Je considère pour ma part que ces deux préfixes doivent être considérés séparément.

${ }^{17}$ Anti possède d'ailleurs une entrée " Nom commun » dans le dictionnaire collaboratif en ligne Wiktionary, avec le sens de "personne opposée ou hostile à un projet» (http://fr.wiktionary.org/wiki/anti), ce qui correspond à l'interprétation adversative du type ANTILIMACES $_{\mathrm{A} / \mathrm{N}}$, pas à l'interprétation antonymique. On notera le marquage du pluriel sur anti- en (17b).

Un relecteur attire toutefois mon attention sur le fait qu'anti, en emploi autonome en tant que nom, n'a pas une autonomie sémantique complète : il y a à chaque fois anaphore (le nom est récupérable en contexte).

${ }^{18} \mathrm{http}: / /$ glossa.fltr.ucl.ac.be/

${ }^{19} \mathrm{http} / / \mathrm{hpsg}$.fu-berlin.de/cow/colibri/

${ }^{20}$ Le TLFi n'a pas une position claire sur la présence du trait d'union dans les formes nominales en non(-) : «le trait d'union est quasi systématique pour les subst.; dans le corpus littér. du TLF, proportion de l'ordre de 1 ou $2 \%$ d'exceptions. Pour les adj., la règle est l'absence du trait d'union, mais il y a des hésitations. Souvent aussi, notamment dans le discours philos., le trait d'union marque une liaison conceptuelle » (TLFi, s.v. non-).

${ }^{21}$ Hathout (2009) range ces dérivés parmi les $[a n t i-\mathrm{N}]_{\mathrm{N}}$ antonymiques (interprétation qu'il appelle « logique », cf. note 1.) ; je choisis d'en faire une catégorie à part étant donné que ces dérivés sont lexicalisés dans un sens bien particulier et qu'ils appartiennent à un vocabulaire spécialisé.

${ }^{22}$ Ce que j'appelle artefacts non intellectuels sont des objets fabriqués physiques non iconiques dans la classification de Flaux \& Van de Velde (2000) ; quant à la distinction entre artefacts informationnels et artefacts artistiques, elle ne recoupe que partiellement celle faite par ces auteurs entre idéalités et objets fabriqués physiques iconiques.

${ }^{23}$ Ces noms qui dénotent des entités sans aucune extension temporelle, caractérisées par une indistinction entre qualité et quantité, et qui "n'existent pas en elles-mêmes, mais seulement par rapport à un sujet » sont des noms abstraits intensifs chez Flaux \& Van de Velde (op. cit., 2000 : 76)

${ }^{24}$ Un relecteur attire mon attention sur la nécessité d'aller au-delà de l'attesté et de m'interroger sur ce qui fait que certains $[\text { non- } \mathrm{N}]_{\mathrm{N}}$ contraires / $[\text { anti- } \mathrm{N}]_{\mathrm{N}}$ antonymiques sont certainement meilleurs ou plus mauvais que d'autres. Cette question est trop complexe pour être abordée en passant et mériterait d'être traitée dans un article séparé. En ce qui concerne les $[n o n-\mathrm{N}]_{\mathrm{N}}$, comme évoqué en introduction, les dérivés peuvent prendre trois interprétations (ontologique, complémentaire, contraire). Une étude sur corpus (en cours) révèle que chacune de ces interprétations a une préférence pour certains types sémantiques de bases : les événements pour les $[n o n-\mathrm{N}]_{\mathrm{N}}$ ontologiques, les êtres humains pour les $[n o n-\mathrm{N}]_{\mathrm{N}}$ complémentaires, les artefacts pour les $[\text { non-N }]_{\mathrm{N}}$ contraires; toutefois, c'est le contexte qui, en dernière instance, orientera le dérivé vers telle ou telle interprétation. D'autre part, comme le remarque le relecteur, il est clair que les bases dénotant des référents potentiellement nuisibles, ou qu'il faut combattre, donneront plus fréquemment lieu à des dérivés $[\text { anti-N }]_{\mathrm{N}}$ adversatifs (du type ANTILIMACES $\mathrm{A}_{\mathrm{N}}$ ) qu'à des $[\text { anti-N }]_{\mathrm{N}}$ antonymiques.

${ }^{25}$ Jonasson emprunte une expression que Wierzbicka (1985) applique aux noms communs.

${ }^{26}$ Même si une requête orientée sur Google ${ }^{T M}$ permet de trouver des $[n o n-\mathrm{N}]_{\mathrm{N}}$ contraires à base NPr référant à des humains, par exemple :

(i) Un non-Mozart qui n'y connait absolument rien en musique, trouvera obligatoirement un accord de quinte (...) très harmonieux, puisque 'mathématiquement' c'est l'accord le plus simple après l'octave. (www)

${ }^{27}$ Type (mais aussi variété) entraîne une lecture taxinomique du nom sur lequel il porte (Flaux \& Van de Velde, $2000: 26)$. 